\title{
Pilomatrix carcinoma: More malignant biological behavior than was considered in the past
}

\author{
MARIOS PAPADAKIS ${ }^{1}$, EELCO DE BREE ${ }^{2}$, NIKOLAOS FLOROS ${ }^{3}$, ELPIDA GIANNIKAKI ${ }^{4}$, \\ ATHINA XEKALOU ${ }^{4}$ and ANDREAS MANIOS ${ }^{2}$
}

\begin{abstract}
${ }^{1}$ Department of Plastic Surgery, Helios Clinic Wuppertal, University Hospital Witten-Herdecke, D-42283 Wuppertal, Germany; ${ }^{2}$ Department of Surgical Oncology, University Hospital of Crete, 71110 Heraklion, Greece; ${ }^{3}$ Department of Surgery, University Hospital Witten-Herdecke, D-42283 Wuppertal, Germany; ${ }^{4}$ Department of Pathology,

General Hospital ‘Venizeleio and Pananio', 71409 Heraklion, Greece
\end{abstract}

Received April 18, 2016; Accepted December 21, 2016

DOI: $10.3892 / \mathrm{mco} .2017 .1148$

\begin{abstract}
Pilomatrix carcinoma is a very rare malignancy, with $\sim 130$ cases reported in the literature. In the past, pilomatrix carcinoma was considered to be a low-grade malignant tumor. Currently, however, its significant recurrence and metastatic potential has been well documented. Lymph node and systemic metastases are frequently observed. Wide surgical excision of the primary lesion is the principal modality of treatment, whereas adjuvant radiotherapy may be beneficial in local tumor control. Lymph node metastases may be treated surgically or with radiotherapy. Systemic disease is not responsive to chemotherapy, and is hence associated with a poor prognosis. Since the majority of nodal and systemic metastases present after the initial diagnosis and treatment, follow-up examinations of these patients may be warranted, despite the currently inadequate treatment options. In the present study, a case of pilomatrix carcinoma of the parotid region with early local recurrence only 2 months after complete excision with negative surgical margins is reported. The local recurrence was treated by excision and radiotherapy. The associated literature is also discussed.
\end{abstract}

\section{Introduction}

Pilomatricoma is a rare benign neoplasm with differentiation towards hair matrix cells, most commonly observed in the head-and-neck region and occurring usually during the first two decades of life. It was first described by Malherbe and Chenantais in 1880 (1), who used the term 'calcifying

Correspondence to: Dr Marios Papadakis, Department of Plastic Surgery, Helios Clinic Wuppertal, University Hospital Witten-Herdecke, Heusnerstr. 40, D-42283 Wuppertal, Germany E-mail: marios_papadakis@yahoo.gr

Key words: pilomatrix carcinoma, malignant pilomatricoma, recurrence, $\beta$-catenin gene epithelioma' to refer to a hard, calcified, benign tumor arising from the sebaceous glands. Dubreuilh and Cazenave reported the hallmark histopathological features of the tumor in 1922 (2), but it was not until 1961 that Forbis and Helwig defined the tumor's origin from the outer root sheath of the hair follicle, suggesting also the word 'pilomatricoma' as a more accurate alternative to Malherbe and Chenantais's term (3).

The locally aggressive malignant equivalent of pilomatricoma, which was first identified by Lopansri and Mihm in 1980 (4), has been referred to as 'pilomatrix carcinoma', 'malignant pilomatricoma', 'trichomatrical carcinoma' or 'calcifying epitheliocarcinoma of Malherbe'. Over 12 cases of pilomatrix carcinoma have been reported (5). This extremely rare tumor has a strong tendency to relapse locally. A high local recurrence rate following simple excision has been reported, and local recurrence may occur even after excision with tumor-free surgical margins $(6,7)$. Although, in the past, this tumor was considered to be a low-grade malignant tumor and unlikely to metastasize, at present its significant metastatic potential has been well documented. Several cases of lymph node metastases have been reported, while in several patients, systemic (mainly pulmonary) metastases occurred (7-11).

In the present report, a case of pilomatrix carcinoma of the parotic region with early local recurrence following complete excision is described. To the best of our knowledge, this is the first report of a pilomatrix carcinoma recurrence occurring only 2 months after resection with negative surgical margins. Diagnostic and therapeutic considerations are discussed.

\section{Case report}

The patient was informed that data regarding her case would be submitted for publication, and she consented.

A 79-year-old woman presented with a hard, solitary, subcutaneous, slow growing mass in the right parotid region (Fig. 1). Clinical examination demonstrated no evidence of lymph node involvement, and the lesion was excised as a benign tumor with a $5 \mathrm{~mm}$ margin. The tumor was intimately associated with the buccal branch of the facial nerve; however, following excision, the function of the 


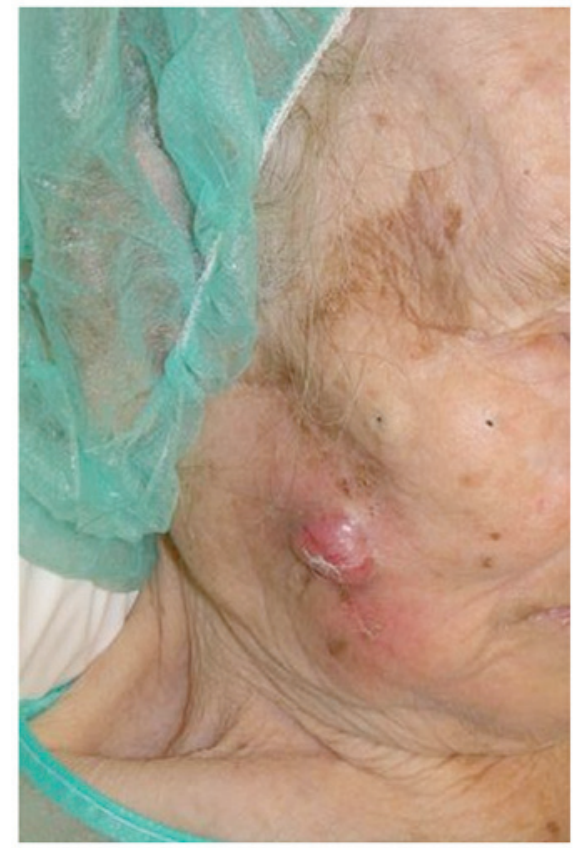

Figure 1. Clinical presentation of the tumor in the right parotid region.

facial nerve remained intact. The postoperative course was uneventful.

On pathological examination, a rather well-circumscribed, dome-shaped, whitish solid tumor, measuring $4 \mathrm{~cm}$ in maximum diameter, was identified in the dermis and subcutaneous tissue of the excised skin. Microscopically, the tumor exhibited the typical features of pilomatrix carcinoma. It was composed of lobules of matrical cells with marked variation in size and shape, and irregular foci of necrosis en masse in the center of several of the lobules (Fig. 2). The tumor cells were composed predominantly of basaloid cells with a variable degree of anaplasia and a high mitotic rate, up to 12 mitotic events/high-power field, as well as atypical mitoses (Fig. 3). In certain areas, the basaloid cells had clear cytoplasm. In the center of several lobules, there was pilar-type keratinization, with the presence of 'ghost' cells, whereas elsewhere, squamous cells were also identified. Peripherally, the tumor cells had invaded the dense fibrous desmoplastic stroma that surrounded the tumor nodules. There was no definite vascular or lymphatic invasion. The epidermis appeared thinner, but remained uninvolved. The tumor was close to the surgical margins, but appeared to have been completely excised. Immunohistochemically, the tumor cells featured cytoplasmic staining with pan-cytokeratin CK14 markers and the monoclonal antibody, Ber-Ep4, nuclear positivity with p63, and focal cytoplasmic staining with carcinoembryonic antigen and CK14, while being negative for CK20, S-100 and the neural cell adhesion molecule, CD56.

Staging of the disease with computed tomography of the neck, chest and abdomen regions was negative for metastatic disease. Despite the tumor-free surgical margins, local recurrence had already occurred 2 months following surgical excision. The patient was reoperated, and subsequently underwent adjuvant radiotherapy. At 4 years after surgical resection

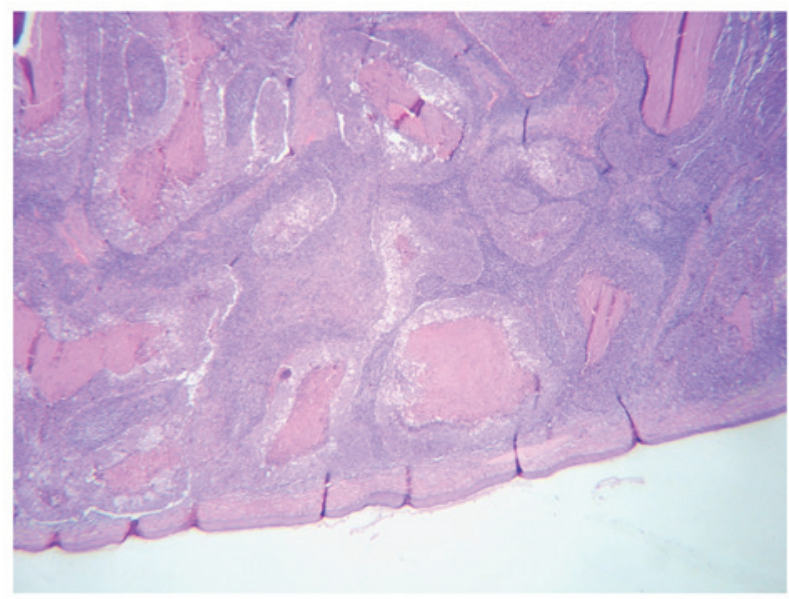

Figure 2. Low-power microscopic view of the pilomatrix carcinoma, consisting of intradermal lobules with basaloid, squamous and clear cells with central necrosis and shadow cells (hematoxylin and eosin staining, x20 magnification).

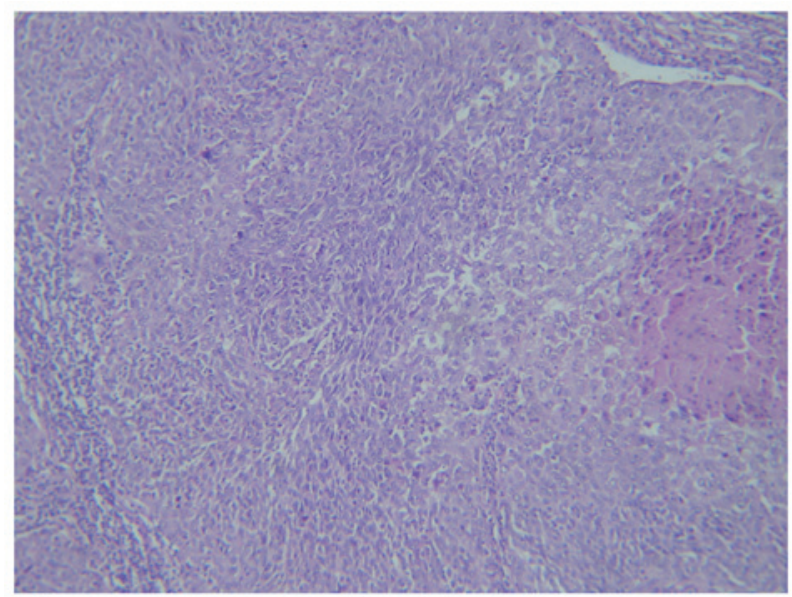

Figure 3. Nests of basaloid tumor cells with cellular atypia and frequent mitoses (hematoxylin and eosin staining, x400 magnification).

of the local recurrence, no further local recurrence or distant metastasis has been observed.

\section{Discussion}

Pilomatrix carcinoma is rare, and in contrast with its benign counterpart, pilomatricoma, has a marked male predominance (male-to-female ratio of 3:1), usually affecting middle-aged adults (mean age of 46 years in a series of 63 cases) (12). It typically presents as a single, hard, firm, painless, asymptomatic, slow growing dermal or subcutaneous mass, occasionally accompanied by bluish skin discoloration or/and ulceration $(13,14)$. A mean size of $4 \mathrm{~cm}$, as was identified in our case, among 60 patients with pilomatrix carcinoma has been reported (12). The majority of the lesions are located in the head-and-neck region, particularly in the preauricular area, scalp, posterior neck and upper back $(12,15,16)$. A very small number of cases have been reported to arise from the parotid region, as in our patient (17). Clinical differential diagnosis includes not only benign pilomatricoma, epidermal cyst, basal 
cell carcinoma and squamous cell carcinoma, but also malignant melanoma and vascular lesions $(12,18)$.

In pilomatrix carcinoma, histology typically reveals irregularly shaped nests of large anaplastic, hyperchromatic basaloid cells with prominent nucleoli, nuclear pleomorphism and abundant mitotic figures, ranging from 8-62/high-power field. Central areas with necrotic debris, shadow cells, clear cytoplasmic cells, transition to squamous cells, invasion of blood and lymphatic vessels, surface ulceration and infiltrative growth pattern are often observed $(13,14,19)$. Follicular matrical differentiation with intratumoral melanocytic hyperplasia has also been observed ('melanocytic matricoma'). Thus, not only cutaneous carcinomas, such as clear cell squamous carcinoma, clear cell porocarcinoma, basal cell carcinoma with matrical differentiation and undifferentiated sebaceous carcinoma, but also malignant melanoma and Merkel's carcinoma should be considered as far as the histological differential diagnosis of pigmented matrical tumors is concerned (18). Although there was no definite vascular or lymphatic invasion, the diagnosis of pilomatrix carcinoma in the present case study was made on the basis of the significant pleomorphism and prominent, multiple nucleoli of the basaloid cells, the number of mitoses, the presence of atypical mitoses, the presence of sizeable areas of necrosis and the infiltrating areas.

Whether pilomatrix carcinoma arises de novo or represents malignant deformation of a pre-existing benign pilomatricoma remains a controversial topic (5). Pilomatrix carcinoma and pilomatricoma are the opposite extremities in the spectrum of hair matrix tumor differentiation, with proliferating pilomatricoma and atypical proliferating pilomatricoma occupying an intermediate place $(8,11)$. Kaddu et al $(8)$ reported a higher probability for local recurrence in incompletely excised proliferating pilomatricoma compared with ordinary pilomatricomas. Benign and malignant pilomatricomas have been associated with mutations in exon 3 of the $\beta$-catenin gene (CTNNB1). Histologically, proliferating pilomatricoma is characterized by lobular aggregations of basaloid cells, slightly atypical nuclei, an expansive growth pattern and lack of vascular or nerve invasion, whereas necrosis is rarely observed (12). The differential diagnosis between pilomatrix carcinoma and proliferating pilomatricoma is often difficult (8). Consequently, the diagnostic accuracy in certain cases reported as being malignant may be questioned (11). Immunohistochemistry is not able to reliably distinguish between benign and malignant hair matrical tumors (20).

Initially, the majority of cases are surgically treated as benign tumors. Local tumor recurrence, as in the present case subject, is observed in the majority of cases in which simple excision is the only treatment, even with the presence of clear surgical margins during conventional histological examination (6). Sau et al (6) reported a recurrence-free interval of 5-17 months following resection in the first large series published (17 patients), whereas Bhasker et al (21) described a recurrence of scalp pilomatrix carcinoma 6 months after resection. Herrmann et al (7) reported, in 2014, three recurrences in a series of 13 cases, 4-13 months following wide local excision. Combining the existing literature with their series, Hermann et al (7) identified a recurrence rate of $23 \%$ in 43 widely excised tumors at an average of 7 months later (median, 6 months) and a recurrence rate of $83 \%$ in 42 simple excised tumors at an average of 11.9 months later (median, 8 months) (7). This yields an overall recurrence rate of 53\%, comparable with the previously published recurrence rate of $60 \%$ from Sau et al (6) in 1993. Due to the high local recurrence rate, wide surgical (re-)excision appears to be indicated. Wide re-excision was omitted, however, for the patient in the present case report, and a conservative policy was advocated due to the patient's wishes and the anatomic location of the tumor.

The definition of adequate surgical margins remains controversial. Several physicians consider a surgical margin of $2 \mathrm{~cm}$ as adequate (22), whereas others, based on their recurrence-free experience, accept a closer surgical margin of $5 \mathrm{~mm}$ (12). Mohs micrographic surgery may provide the optimal treatment for this tumor, given its ability to have complete histological margin control (23). Lymph node metastases should be treated with regional lymph node dissection, when possible. In view of the high possibility of lymph node metastases $(9-11,24)$, sentinel node biopsy could be considered. However, the option of sentinel node biopsy is moderated by the fact that, in certain of these cases, lymph node involvement is already clinically confirmed at the initial presentation. Additionally, in over half of the patients with nodal metastases, systemic metastases are concurrently identified $(9,10)$. Since systemic disease determines the prognosis, the additional value of sentinel node biopsy and early removal of non-palpable lymph node metastases is diminished.

Only rarely are systemic metastases present at diagnosis (24). The presence of local recurrence and tumor location on the extremities is considered to increase the metastatic potential of malignant pilomatricoma, although in 5 cases, metastasis occurred at the time of initial presentation or in the absence of clinical recurrence (7). In the majority of cases, however, metastases are observed as recurrent disease, with a median time from diagnosis to metastases of 18 months, and a median survival following diagnosis of metastases of 16 months (13). Administration of various chemotherapeutic agents has not proven to be effective $(9,13)$.

The role of radiotherapy in the treatment of this tumor has yet to be fully elucidated. However, adjuvant radiotherapy following excision of primary and recurrent tumors, as applied in the present case, has been reported to provide adequate local tumor control (9). Furthermore, radiotherapy has been considered to be a good alternative in patients for whom adequate excision is not possible (12). Radiotherapy may also be an alternative to formal surgical dissection of involved lymph nodes, and provide palliative treatment of systemic metastases $(9,13)$.

In conclusion, in the present case study an early recurrent pilomatrix carcinoma of the parotic region has been reported. Pilomatrix carcinoma should always be considered in the differential diagnosis of hard solitary tumors of the head-and-neck region, as its recurrence rate following simple excision remains considerably high. Lymph node and systemic metastases are also observed in $\sim 13 \%$ of the reported cases (7). Wide surgical excision of the primary lesion is the principal modality of treatment, and should be considered as the preferred option. Adjuvant radiotherapy may be beneficial in local tumor control. Lymph node metastases may be treated surgically, or with radiotherapy. Systemic disease is not 
responsive to chemotherapy, and is hence associated with a poor prognosis. Since the majority of nodal and systemic metastases present following initial diagnosis and treatment, follow-up examinations of these patients may be warranted, despite the currently inadequate treatment options.

\section{Acknowledgements}

We would like to thank Mrs. Tzeni Bolbasis for linguistically reviewing the manuscript.

\section{References}

1. Malherbe A and Chenantais J: Sur l'epithelioma calcifie des glandes sebacees. Progr Med 8: 826-828, 1880.

2. Dubreuilh W and Cazenave E: De l'epithelioma calcifie: Etude histologique. Ann Dermatol Syphilol 3: 257-268, 1922.

3. Forbis $\mathrm{R} \mathrm{Jr}$ and Helwig EB: Pilomatrixoma (calcifying epithelioma). Arch Dermatol 83: 606-618, 1961.

4. Lopansri S and Mihm MC Jr: Pilomatrix carcinoma or calcifying epitheliocarcinoma of Malherbe: A case report and review of literature. Cancer 45: 2368-2373, 1980.

5. Nishioka M, Tanemura A, Yamanaka T, Tani M, Miura H, Asakura M, Tamai N and Katayama I: Pilomatrix carcinoma arising from pilomatricoma after 10-year senescent period: Immunohistochemical analysis. J Dermatol 37: 735-739, 2010.

6. Sau P, Lupton GP and Graham JH: Pilomatrix carcinoma. Cancer 71: 2491-2498, 1993.

7. Herrmann JL, Allan A, Trapp KM and Morgan MB: Pilomatrix carcinoma: 13 new cases and review of the literature with emphasis on predictors of metastasis. J Am Acad Dermatol 71: 38-43.e2, 2014.

8. Kaddu S, Soyer HP, Wolf IH and Kerl H: Proliferating pilomatricoma. A histopathologic simulator of matrical carcinoma. J Cutan Pathol 24: 228-234, 1997.

9. Tselis N, Heyd R, Vogt HG and Zamboglou N: Pilomatrix carcinoma with lymph node and pulmonary metastases. Strahlenther Onkol 182: 727-732, 2006.

10. Bassarova A, Nesland JM, Sedloev T, Danielsen H and Christova S: Pilomatrix carcinoma with lymph node metastases. J Cutan Pathol 31: 330-335, 2004.
11. Autelitano L, Biglioli F, Migliori G and Colletti G: Pilomatrix carcinoma with visceral metastases: Case report and review of the literature. J Plast Reconstr Aesthet Surg 62: e574-e577, 2009.

12. Hardisson D, Linares MD, Cuevas-Santos J and Contreras F: Pilomatrix carcinoma: A clinicopathologic study of six cases and review of the literature. Am J Dermatopathol 23: 394-401, 2001.

13. Mikhaeel NG and Spittle MF: Malignant pilomatrixoma with multiple local recurrences and distant metastases: A case report and review of the literature. Clin Oncol (R Coll Radiol) 13: 386-389, 2001.

14. Lan MY, Lan MC, Ho CY, Li WY and Lin CZ: Pilomatricoma of the head and neck: A retrospective review of 179 cases. Arch Otolaryngol Head Neck Surg 129: 1327-1330, 2003.

15. Manivel C, Wick MR and Mukai K: Pilomatrix carcinoma: An immunohistochemical comparison with benign pilomatrixoma and other benign cutaneous lesions of pilar origin. J Cutan Pathol 13: 22-29, 1986.

16. Waxtein L, Vega E, Alvarez L, Cortes-Franco R, Hojyo T and Dominguez-Soto L: Malignant pilomatricoma: A case report. Int J Dermatol 37: 538-540, 1998.

17. Liu JF, Li B, Fan ZX, Jiao T, Li C, Qin S, Lang JY and Chen JX: Pilomatrix carcinoma on the left side of the parotid region: A case report and review of the literature. Oncol Lett 10: 313-316, 2015.

18. Jani P, Chetty R and Ghazarian DM: An unusual composite pilomatrix carcinoma with intralesional melanocytes: Differential diagnosis, immunohistochemical evaluation, and review of the literature. Am J Dermatopathol 30: 174-177, 2008.

19. Scheinfeld N: Pilomatrical carcinoma: A case in a patient with HIV and hepatitis C. Dermatol Online J 14: 4, 2008.

20. Kondo T and Tanaka Y: Malignant pilomatricoma in the parietal area. Pathol Oncol Res 12: 251-253, 2006.

21. Bhasker S, Bajpai V, Bahl A and Kalyanakuppam S: Recurrent pilomatrix carcinoma of scalp treated by electron beam radiation therapy. Indian J Cancer 47: 217-219, 2010.

22. Fujiwara $T$, Yamamoto $H$ and Hashiro M: Malignant pilomatricoma. Scand J Plast Reconstr Surg Hand Surg 36: 119-121, 2002.

23. Sable D and Snow SN: Pilomatrix carcinoma of the back treated by mohs micrographic surgery. Dermatol Surg 30: 1174-1176, 2004.

24. Tawfiq N, Lakhrib N, Mharech A, Benchakroun N, Benider A, Benkirane A, Zamiati S, Mansouri I, Roubal M, Kadiri Fatmi ME, et al: Malignant pilomatrixoma of head and neck. A case report. Cancer Radiother 14: 198-201, 2010 (In French). 